\title{
Selective spleen tyrosine kinase inhibition delays autoimmune arthritis in mice
}

\author{
ZUOFU ZHANG ${ }^{1,2 *}, \mathrm{CHUNNI} \mathrm{CAO}^{3 *}, \mathrm{SHUI} \mathrm{SUN}^{1}$ and QIANG XU ${ }^{2}$ \\ ${ }^{1}$ Department of Joint Surgery, Shandong Provincial Hospital, Shandong University, Jinan, Shandong 250021; \\ Departments of ${ }^{2}$ Joint Surgery and ${ }^{3}$ Hyperbaric Oxygen, Yantai Yuhuangding Hospital, Yantai, Shandong 264000, P.R. China
}

Received July 8,2014; Accepted April 10, 2015

DOI: $10.3892 / \mathrm{mmr} .2015 .3759$

\begin{abstract}
Spleen tyrosine kinase (SYK) has an important role in immunoreceptor signaling, and SYK inhibition has accordingly attenuated immune-mediated injury in several models. Therefore, the present study examined the effect of SYK inhibition with the selective spleen tyrosine kinase inhibitor P505-15 in experimental rheumatoid arthritis (RA) using a murine model of collagen-induced arthritis (CIA). Treatment with the selective SYK inhibitor P505-15, a small molecule kinase inhibitor selective for SYK, led to a reduction in arthritis score and attenuated histological damage. P505-15 reduced cartilage destruction and macrophage infiltration in CIA mice. In addition, P505-15-treated mice showed lower circulating levels of type II-collagen immunoglobulin (Ig)G1 and IgG2 and pro-inflammatory cytokines. Importantly, P505-15 treatment markedly reduced the interleukin $1 \beta$-stimulated inflammatory response in human RA synovial cells. Given these encouraging results, a key function for SYK in the development of RA was identified, highlighting that SYK may be a potential therapeutic target for human RA.
\end{abstract}

\section{Introduction}

Rheumatoid arthritis (RA) is a systemic autoimmune disease characterized by the hyperplasia of synovial tissue and progressive destruction of articular cartilage, bone and ligaments (1-2). The pathogenesis of RA is a complex process mediated by an interdependent network of cytokines, proteolytic enzymes and prostanoids (3). There thus remains a tremendous unmet

Correspondence to: $\mathrm{Dr}$ Shui Sun, Department of Joint Surgery, Shandong Provincial Hospital, Shandong University, 324 Jing-5-Wei-7 Road, Jinan, Shandong 250021, P.R. China

E-mail: sunshuisd@163.com

${ }^{*}$ Contributed equally

Key words: spleen tyrosine kinase, inhibitor, autoimmune arthritis, collagen-induced arthritis clinical requirement for more effective therapeutic strategies, with a goal of sustained remission for a greater number of patients with RA.

Current therapeutic strategies pursued by the biopharmaceutical industry include those that target the key kinases involved in the pathogenesis of RA. One such enzyme is the spleen tyrosine kinase (SYK), which is a master regulator in coupling activated immunoreceptors to the mobilization of downstream signal transduction cascades that affect diverse biological functions (4). Given the central role of SYK in transmission of antigen receptor signals that are critical for autoantibody production and the various innate immune effector functions, pharmacological inhibition of the catalytic function of SYK is expected to have pleiotropic anti-inflammatory effects in the pathogenesis of autoimmune disorders (5).

The present study explored whether the selective SYK inhibitor P505-15 impacted the development of collagen-induced arthritis (CIA) disease in vivo in mice. The results of the present study indicated that P505-15 may serve as a lead candidate for further development of selective SYK inhibitors for the potential treatment of RA.

\section{Materials and methods}

Animals. A total of 24 male DBA1/J mice (28-30 g; 8 weeks old) were obtained from Charles River Breeding Laboratories (Wilmington, MA, USA). All animal experiments were approved by the Institutional Animal Use Committee of Shandong University (Jinan, China). The study was approved by the ethics committee of Shandong Provincial Hospital, Shandong University (no. G2015698). The mice were housed with a 12-h light/dark cycle. They had ad libitum access to food and water in cages $(n=6)$ under controlled temperature and humidity.

CIA induction. To induce CIA in DBA1/J mice as previously described (6), type II collagen (CII; Chondrex, Redmond, WA, USA) was dissolved overnight in $0.1 \mathrm{~N}$ acetic acid $(4 \mathrm{mg} / \mathrm{ml}$; (Chondrex) with gentle rotation at $4^{\circ} \mathrm{C}$. The mice were injected intradermally at the base of the tail with $100 \mu \mathrm{g}$ CII emulsified 1:1 in complete Freund's adjuvant (Chondrex) and then received a second injection with $100 \mu \mathrm{g}$ CII emulsified 1:1 in incomplete Freund's adjuvant 21 days later. 
Assessment of arthritis. Clinical arthritis scores were evaluated using a scale of 0-4 for each limb as previously described (7). Hind paw thickness was measured with an electric caliper placed across the ankle joint at the widest point. The paw thickness index was defined as the percent increase in the diameter of the arthritic ankle at specific time-points as compared with the diameter determined on day 21. On day 38 , the mice were sacrificed, and joint tissue samples from each animal were harvested for end-point histological analysis.

Administration of P505-15. On day 24 after the first immunization, CIA mice ( $\mathrm{n}=12$ per group) received oral doses of vehicle $(0.5 \%$ methylcellulose in water) or P505-15 $(15 \mathrm{mg} / \mathrm{kg})$ twice daily for 14 days as previously reported (8). On day 38, mice were anesthetized by subcutaneous injection of ketamine (Fort Dodge Animal Health, Webster County, IN, USA) and exsanguinated via cardiac puncture. Joints were stained with hematoxylin and eosin (H\&E) for histology.

Histological assessment. Mouse joint tissue specimens from mice with CIA were fixed with $10 \%$ formalin, decalcified for 15 days in $10 \%$ EDTA, dehydrated and embedded in paraffin. Sections $(6 \mu \mathrm{m})$ were stained with $\mathrm{H} \& \mathrm{E}$ and Safranin O-fast green for light microscopy (Labophot-2; Nikon, Tokyo, Japan). The mouse joint sections were scored for changes in synovial inflammation and bone erosion on a scale of 0-4 as described in the previous literature (7). Rabbit anti-mouse monoclonal anti-CD68 antibody (1:2,000 dilution; cat. no. 76308; Cell Signaling Technology, Beverly, MA, USA) incubated for $2 \mathrm{~h}$ at $37^{\circ} \mathrm{C}$ was used to identify the synovial macrophages. Safranin O-fast green staining was scored 0-3 (7). For immunohistochemical staining, expression of CD68 in the synovial tissue of all ankle joints present was scored semiquantitatively on a five-point scale (9). A score of 0 represented minimal expression, while a score of 4 represented abundant expression of a marker.

Assessment of anti-CII antibodies and cytokines. Sera were obtained from anesthetized animals by retroorbital puncture at the end of the study. Serum levels of anti-CII immunoglobulin (Ig)G1 and -IgG2a were measured by ELISA (Chondrex, Redmond, WA, USA). The levels of tumor necrosis factor (TNF)- $\alpha$, interleukin (IL)-6 and IL-17 were measured by TNF- $\alpha$, IL-6 and IL-17 ELISA kits (R\&D Systems, Minneapolis, MN, USA) according to the manufacturer's instructions.

Human RA synovial cells. Synovial tissue from 10 RA patients was digested with $0.5 \mathrm{mg} / \mathrm{ml}$ collagenase A in RPMI medium for $2 \mathrm{~h}$ at $37^{\circ} \mathrm{C}$. The cells were washed twice with $10 \%$ phosphate-buffered saline/Dulbecco's modified Eagle's medium and filtered using a $0.70-\mu \mathrm{m}$ cell strainer (Falcon, BD Biosciences, Franklin Lakes, NJ, USA). The cells were washed and $3 \times 10^{6}$ cells were plated in six-well plates. After overnight incubation, the cells were treated with the selective SYK inhibitor P505-15 (100 $\mu \mathrm{mol} / \mathrm{l})$ for $48 \mathrm{~h}$. The cells were then treated with $20 \mathrm{ng} / \mathrm{ml}$ IL-1 $\beta$ (R\&D Systems) for $4 \mathrm{~h}$. The cytokines in the cell supernatants were quantified by ELISA. Results are expressed as the average percent of the values in untreated controls. Written consent was obtained from all patients and all experiments were approved by the Institutional Ethics Committee of Shandong University (Jinan, China).

Statistical analysis. Values are expressed as the mean \pm standard error of the mean. Statistical analyses were performed using SPSS 16.0 (SPSS, Inc., Chicago, IL, USA). Statistical comparisons were performed using one-way analysis of variance. The significance of differences between groups was determined using Student's unpaired $t$-test. $\mathrm{P}<0.05$ was considered to indicate a significant difference between values.

\section{Results}

P505-15 treatment attenuates arthritis score and joint damage in CIA mice. The mice started developing arthritis 24 days after the first immunization. Clinical scores of CIA in DBA1/J mice were recorded every 2 days from 24 days after the first immunization. P505-15 treatment significantly attenuated the clinical symptoms of arthritis from day 32 onwards (Fig. 1A). H\&E staining (Fig. 1B) showed that the joints of vehicle-treated CIA mice showed marked infiltration of leukocytes and bone damage. In the joints of P505-15-treated CIA mice, the infiltration of inflammatory cells and bone erosion were significantly inhibited (Fig. 1B).

P505-15 reduces cartilage destruction and macrophage infiltration in CIA mice. In addition, the present study investigated the efficacy of P505-15 treatment in CIA bone erosion using safranin-O staining. The results indicated that the joints of CIA mice showed evident cartilage destruction, which was significantly reduced in the CIA mice treated with P505-15 (Fig. 2A and B).

The synovial tissue sections were also assessed for the expression of CD68 using immunohistochemistry. A significant reduction in CD68 expression was observed in the CIA mice treated with P505-15 compared with that in the control group $(\mathrm{P}<0.01$; Fig. $2 \mathrm{C}$ and $\mathrm{D})$.

P505-15 treatment inhibits anti-CII IgG1, -IgG2a and pro-inflammatory cytokines in CIA mice. The effects of P505-15 administration on the serum anti-CII IgG1 and -IgG2a were assessed. The results indicated that P505-15 treatment significantly reduced the number of anti-CII antibodies relative to that in vehicle-treated CIA controls ( $\mathrm{P}<0.01$; Fig. 3A). CIA is characterized by marked expression of pro-inflammatory cytokines (10). To ascertain whether P505-15 inhibits this characteristic, P505-15-treated and -untreated mice with CIA were bled on day 38. Serum TNF- $\alpha$, IL-6 and IL-17 levels were measured using an ELISA (Fig. 3B). The levels of these pro-inflammatory cytokines were markedly increased in the vehicle-treated group compared with those in the normal group, which was significantly attenuated by treatment with P505-15 ( $\mathrm{P}<0.01)$. The results therefore suggested that P505-15 may have a therapeutic effect to reduce the severity of CIA by inhibiting the production of inflammatory cytokines.

P505-15 treatment significantly decreases inflammation in human RA synovial cells. To assess whether a similar phenomenon to that in the mouse model of CIA can be observed in 

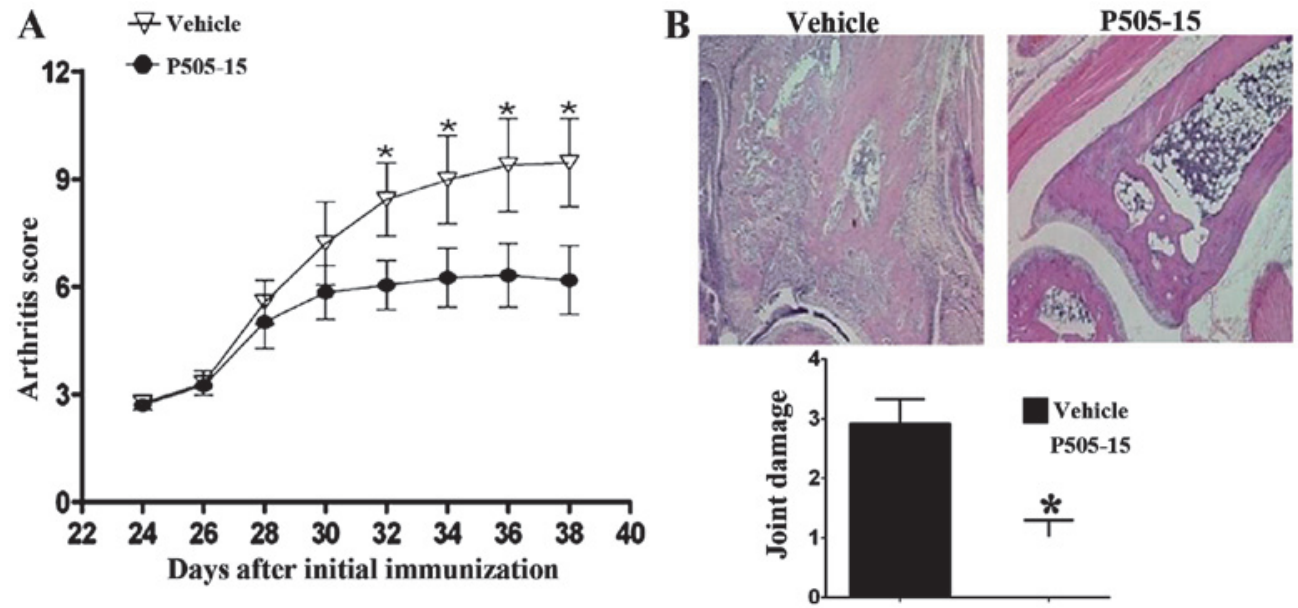

Figure 1. P505-15 treatment reduces the severity of arthritis and histology. (A) Clinical score was assessed every 48 h. (B) hematoxylin and eosin staining of vehicle-treated CIA mice, and P505-15-treated CIA mice, with histological scoring. CIA mice were treated with P505-15 or control, starting at 24 days after initial immunization (magnification, $\mathrm{x} 100$ ). Values are expressed as the mean \pm standard error of the mean of 12 mice per group. ${ }^{*} \mathrm{P}<0.01$ vs control group. CIA, collagen-induced arthritis.
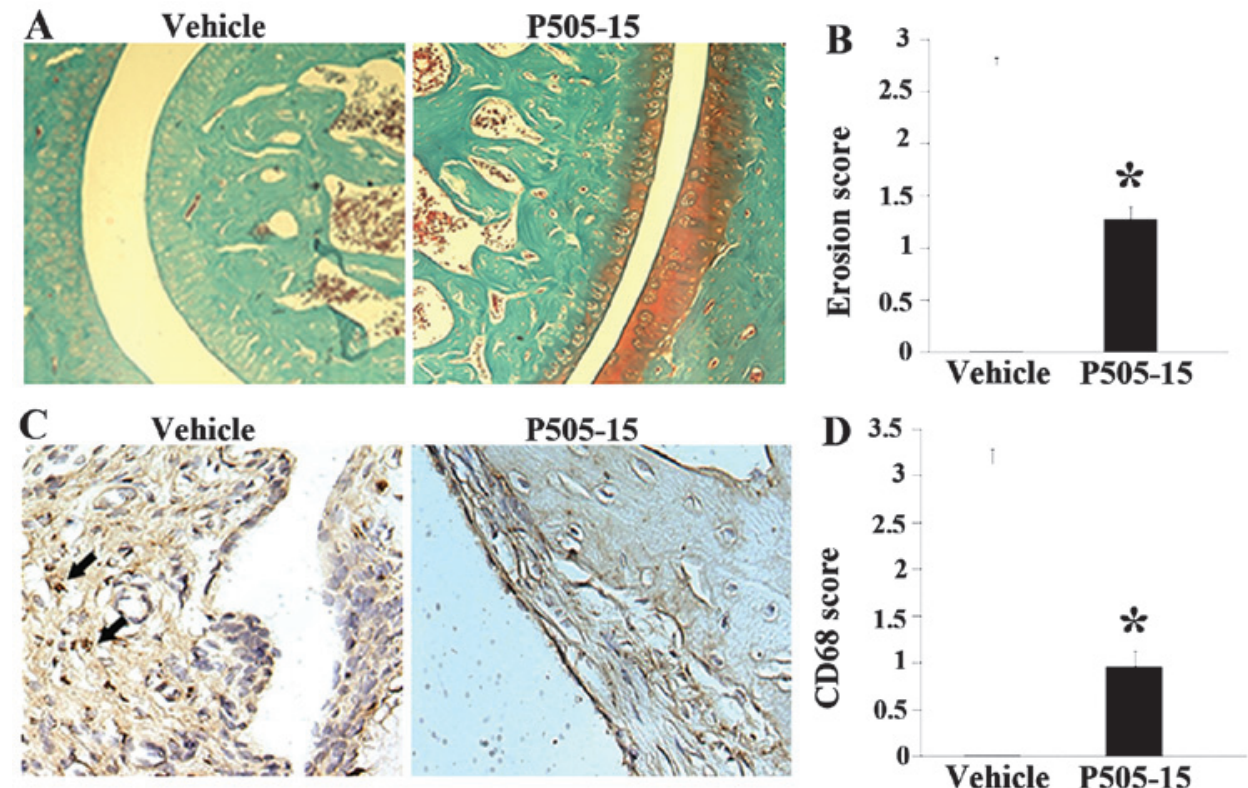

Figure 2. P505-15 attenuates cartilage destruction and macrophage infiltration in CIA mice. (A) Representative histopathology images of the ankle joints stained with Safranin-O (magnification, x100). (B) Histological evaluation of cartilage erosion in CIA mice after P505-15 therapy. (C) Immunohistochemical analysis indicated that P505-15 inhibited the expression of CD68 in synovial tissue of CIA mice (magnification, x100). (D) Quantified CD68 expression in CIA mice. Values are expressed as the mean \pm standard error of the mean of 12 mice per group. ${ }^{*} \mathrm{P}<0.01$ vs control group. CIA, collagen-induced arthritis.
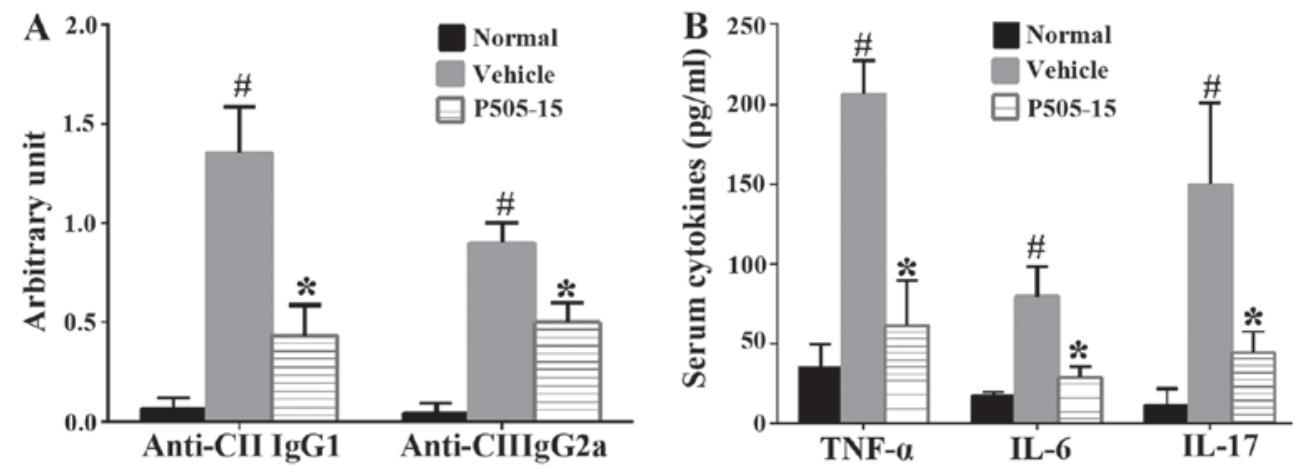

Figure 3. P505-15 treatment inhibits the production of (A) autoantibodies and (B) pro-inflammatory cytokines in sera from CIA mice. On day 38, sera were collected and various cytokines were measured by ELISA. Values are expressed as the mean \pm standard error of the mean of 12 mice per group. "P<0.01 vs. normal mice; "P<0.01 vs. vehicle-treated group. CII, collagen type II; CIA, collagen-induced arthritis; TNF, tumor necrosis factor; IL, interleukin; Ig, immunoglobulin. 


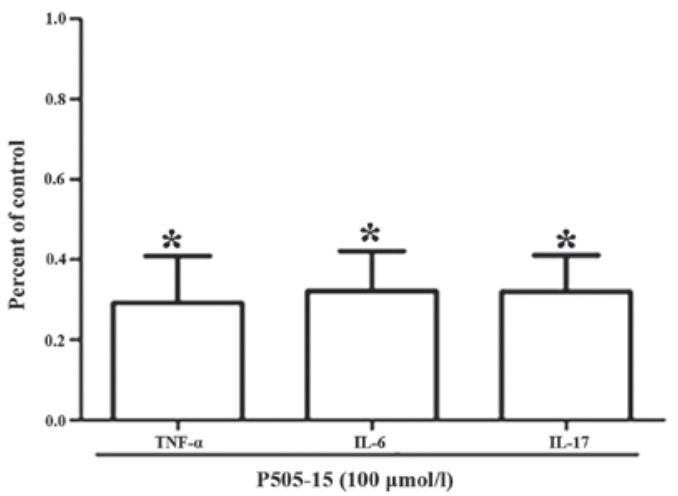

Figure 4. P505-15 treatment inhibits inflammation in human rheumatoid arthritis synovial cells. Cells were treated with P505-15 (100 $\mu \mathrm{mol} / \mathrm{ml})$ for 48 h. P505-15 decreased the levels of TNF- $\alpha$, IL-6, and IL-17 in synovial cell cultures. Cytokine release is expressed as the average percentage of the values in control-treated controls/(percent of control cells in the absence of P505-15). Values are expressed as the mean \pm standard error of the mean of 10 patients. " $\mathrm{P}<0.01$ vs. control cells in the absence of P505-15. TNF, tumor necrosis factor; IL, interleukin.

human RA, RA synovial cells were cultured in the presence or absence of the SYK inhibitor P505-15 (100 $\mu \mathrm{mol} / \mathrm{l})$ for $48 \mathrm{~h}$. The cells were then treated with $20 \mathrm{ng} / \mathrm{ml} \mathrm{IL-1 \beta}$ (R\&D Systems) for $4 \mathrm{~h}$. Supernatants were then assayed for TNF- $\alpha$, IL-6 and IL-17. The results indicated that P505-15 treatment significantly reduced the levels of TNF- $\alpha$, IL- 6 and IL-17 (Fig. 4). The results therefore suggested that SYK inhibition attenuates experimental arthritis via inhibiting the generation of a pro-inflammatory response.

\section{Discussion}

The results of the present study suggested that SYK warrants clinical investigation as a therapeutic target in autoimmune arthritis. In addition to the known anti-inflammatory effects of SYK (11), the present study was the first, to the best of our knowledge, to show that SYK inhibition has a significant impact on CIA using a highly accurate pre-clinical autoimmune arthritis model. More importantly, the present study reported the effects of SYK inhibition in human RA synovial cells.

Pharmacological inhibition of SYK using fostamatinib or other small molecule inhibitors as potential immunomodulatory agents is being actively pursued for the treatment of autoimmune and inflammatory disorders in a number of in vivo models of immune-mediated injury $(5,11,12)$. However, to the best of our knowledge, P505-15, a highly specific and potent inhibitor of purified SYK, has not been tested in rheumatoid arthritis models. SYK is a protein tyrosine kinase that couples B-cell receptor (BCR) activation with downstream signaling pathways, affecting cell survival and proliferation (13). P505-15 has been shown to inhibit BCR-dependent secretion of the chemokines CCL3 and CCL4 by CLL cells, and leukemia cell migration toward the tissue homing chemokines CXCL12, CXCL13, and beneath stromal cells, which demonstrates that the selective SYK inhibitor P505-15 is highly effective in the inhibition of CLL survival and tissue homing circuits, and supports the therapeutic development of these agents in patients with CLL, other B-cell malignancies and autoimmune disorders (13). The present study implicated SYK in the pathogenesis of RA and demonstrated that P505-15 is highly effective for ameliorating CIA disease. These results are also supported by a study showing that genetic deficiency of SYK in the hematopoietic compartment completely blocked the development of arthritis and also prevented the appearance of periarticular bone erosions (14). In further support of the results of the present study, it was reported that SYK inhibition suppresses the development of lupus disease and ameliorates established disease in lupus-prone mice, and may therefore represent a valuable treatment for patients with systemic lupus erythematosus $(15,16)$.

In addition to preventing the production of pathogenic autoantibodies, including serum anti-CII IgG1 and -IgG2a, the present study observed that the pro-inflammatory cytokine production in CIA was inhibited by P505-15. In addition, SYK has been reported to have a crucial role in macrophage activation $(17,18)$. Consistent with this observation, the present study showed that blockade of intracellular SYK inhibited macrophage activation. Strengths of the present study, however, include the use of a genuine autoimmune model of RA using a well characterized selective SYK inhibitor. The striking findings of the present study using this experimental model suggested that clinical studies on SYK as a therapeutic target in RA are desirable.

\section{References}

1. Lee HS, Woo SJ, Koh HW, Ka SO, Zhou L, Jang KY, Lim HS, Kim HO, Lee SI and Park BH: Regulation of apoptosis and inflammatory responses by insulin-like growth factor binding protein 3 in fibroblast-like synoviocytes and experimental animal models of rheumatoid arthritis. Arthritis Rheumatol 66: 863-873, 2014.

2. Firestein GS: Evolving concepts of rheumatoid arthritis. Nature 423: 356-361, 2003.

3. Meier FM, Frerix M, Hermann W and Müller-Ladner U: Current immunotherapy in rheumatoid arthritis. Immunotherapy 5: 955-974, 2013.

4. Riccaboni M, Bianchi I and Petrillo P: Spleen tyrosine kinases: biology, therapeutic targets and drugs. Drug Discov Today 15: 517-530, 2010.

5. Tan S, Liao C, Lucas C, Stevenson C and DeMartino JA: Targeting the SYK-BTK axis for the treatment of immunological and hematological disorders: recent progress and therapeutic perspectives. Pharmacol Ther 138: 294-309, 2013.

6. Moon SJ, Park JS, Woo YJ, Lim MA, Kim SM, Lee SY, Kim EK, Lee HJ, Lee WS, Park SH, Jeong JH, et al: Rebamipide suppresses collagen-induced arthritis through reciprocal regulation of th17/treg cell differentiation and heme oxygenase 1 induction. Arthritis Rheumatol 66: 874-885, 2014.

7. Criado G, Risco A, Alsina-Beauchamp D, Pérez-Lorenzo MJ, Escós A and Cuenda A: Alternative p38 MAPKs are essential for collagen-induced arthritis. Arthritis Rheumatol 66: 1208-1217, 2014.

8. Spurgeon SE, Coffey G, Fletcher LB, Burke R, Tyner JW, Druker BJ, Betz A, DeGuzman F, Pak Y, Baker D, Pandey A, et al: The selective SYK inhibitor P505-15 (PRT062607) inhibits B cell signaling and function in vitro and in vivo and augments the activity of fludarabine in chronic lymphocytic leukemia. J Pharmacol Exp Ther 344: 378-387, 2013.

9. Tak PP, Smeets TJ, Daha MR, Kluin PM, Meijers KA, Brand R, Meinders AE and Breedveld FC: Analysis of the synovial cell infiltrate in early rheumatoid synovial tissue in relation to local disease activity. Arthritis Rheum 40: 217-225, 1997.

10. Souza PP and Lerner UH: The role of cytokines in inflammatory bone loss. Immunol Invest 42: 555-622, 2013.

11. Kaur M, Singh M and Silakari O: Inhibitors of switch kinase 'spleen tyrosine kinase' in inflammation and immune-mediated disorders: a review. Eur J Med Chem 67: 434-446, 2013. 
12. Ghosh D and Tsokos GC: Spleen tyrosine kinase: an Src family of non-receptor kinase has multiple functions and represents a valuable therapeutic target in the treatment of autoimmune and inflammatory diseases. Autoimmunity 43: 48-55, 2010.

13. Hoellenriegel J, Coffey GP, Sinha U, Pandey A, Sivina M, Ferrajoli A, Ravandi F, Wierda WG, O'Brien S, Keating MJ and Burger JA: Selective, novel spleen tyrosine kinase (Syk) inhibitors suppress chronic lymphocytic leukemia B-cell activation and migration. Leukemia 26: 1576-1583, 2012.

14. Jakus Z, Simon E, Balázs B and Mócsai A: Genetic deficiency of Syk protects mice from autoantibody-induced arthritis. Arthritis Rheum 62: 1899-1910, 2010.

15. Chauhan AK and Moore TL: Immune complexes and late complement proteins trigger activation of Syk tyrosine kinase in human CD4(+) T cells. Clin Exp Immunol. 167: 235-245, 2012.
16. Deng GM, Liu L, Bahjat FR, Pine PR and Tsokos GC: Suppression of skin and kidney disease by inhibition of spleen tyrosine kinase in lupus-prone mice. Arthritis Rheum 62: 2086-2092, 2010.

17. Ulanova M, Asfaha S, Stenton G, Lint A, Gilbertson D, Schreiber A and Befus D: Involvement of Syk protein tyrosine kinase in LPS-induced responses in macrophages. J Endotoxin Res 13: 117-125, 2007.

18. Stenton GR, Ulanova M, Déry RE, Merani S, Kim MK, Gilchrist M, Puttagunta L, Musat-Marcu S, James D Schreiber AD and Befus AD: Inhibition of allergic inflammation in the airways using aerosolized antisense to Syk kinase. J Immunol 169: 1028-1036, 2002. 